\title{
COVID-19 perinatal en América Latina
}

\author{
Augusto Sola ${ }^{1}$, Susana Rodríguez², Marcelo Cardetti ${ }^{3}$, Carmen Dávila ${ }^{4}$
}

Forma de citar

Sola A, Rodríguez S, Cardetti M y Dávila C. COVID-19 perinatal en América Latina. Rev Panam Salud Publica. 2020;44e:47. https://doi.org/10.26633/RPSP.2020.47

RESUMEN

Objetivo. Evaluar y reportar las características clínicas y los resultados de la infección por SARS-CoV-2 en mujeres embarazadas y recién nacidos en América Latina.

Métodos. Estudio descriptivo basado en el reporte prospectivo de las unidades constituyentes de la Red de la Sociedad Iberoamericana de Neonatología.

Resultados. De 86 mujeres embarazadas con COVID-19 confirmadas por RT-PCR en siete países (6 de América Latina y Guinea Ecuatorial) 68\% (59) fueron asintomáticas. Del 32\% de mujeres sintomáticas, 89\% (24) tuvieron síntomas leves y 3,5\% (3) presentaron síntomas respiratorios graves. Ninguna mujer falleció. La tasa de cesáreas fue de 38\%; la edad gestacional fue $<37$ semanas en $6 \%$ de los casos. Se realizó RT-PCR a todos los recién nacidos (RN) entre las 16 y 36 horas de vida; en $6(7 \%)$ el resultado del hisopado fue positivo. Todos ellos presentaron dificultad respiratoria leve y transitoria; ninguno falleció. Dos RN con RT-PCR negativa fallecieron por otras causas. Se autorizó el amamantamiento en solo $24 \%$ de las madres; en $13 \%$ se extrajo leche y en $63 \%$ se alimentó al RN con fórmula. En $76 \%$ de los casos se separó al binomio madre-hijo, y en $95 \%$ de los casos la madre no pudo ser acompañada en el parto ni el puerperio.

Conclusiones. Son preocupantes la falta de acompañamiento materno, la baja tasa de lactancia y la frecuente separación de la díada madre-hijo. El equipo de salud debe reflexionar sobre la necesidad de defender el cuidado humanizado y centrado en la familia durante esta pandemia.

Palabras clave Infecciones por coronavirus; pandemias; enfermedades del recién nacido; transmisión vertical de enfermedad infecciosa; América Latina.

La nueva infección por coronavirus ha ocasionado una epidemia que se ha expandido rápidamente para convertirse en una de las amenazas de salud más importante de los últimos tiempos ${ }^{1}$. El coronavirus 2 del síndrome respiratorio agudo severo (SARS-CoV-2) causa la enfermedad por coronavirus 2019 (COVID-19).

Se ha ido aprendiendo progresivamente sobre el cuidado general en adultos, pero no así sobre la COVID-19 en la mujer embarazada y el recién nacido (RN). Por lo tanto, es necesario ser prudente al formular recomendaciones y evitar hacerlas sin evidencia firme ${ }^{2}$. Esta evidencia está en construcción y plantea desafíos importantes para crear un ambiente seguro para los pacientes y los agentes de salud perinatal. Es imprescindible que esta situación no atente contra los estándares de cuidado ético y compasivo durante el embarazo, el nacimiento y la etapa neonatal, períodos de máxima vulnerabilidad en la vida ${ }^{3,4}$.

Hasta el momento el SARS-CoV-2 ha causado pocos casos de COVID-19 en RN y mujeres embarazadas; en los casos reportados el cuadro clínico con frecuencia ha sido leve $e^{5,6}$.

Aunque existen publicaciones sobre COVID-19 en mujeres embarazadas y RN, todas informan experiencias en uno o dos centros, ninguna incluye un gran número de $\operatorname{casos}^{5-8} \mathrm{y}$ ninguna proviene de América Latina.

Hasta el momento los tratamientos en evaluación, como antivirales, corticosteroides, plasma de convaleciente o antiparasitarios, no han demostrado efectividad ni seguridad y por lo

\footnotetext{
Sociedad Iberoamericana de Neonatología, Wellington, Estados Unidos de América; ORCID 0000-0002-7608-3872

2 Sociedad Iberoamericana de Neonatología, Buenos Aires, Argentina; ORCID 0000-0001-6015-6048 $\bowtie$ Susana Rodríguez, susana.rodriguez@siben.net
} 
tanto no deberían ser empleados fuera de protocolos de investigación. Por otro lado, el uso de oxígeno durante el parto no solo es innecesario si la oximetría de pulso de la madre es normal, sino que puede generar riesgo de aerosolización y transmisión del virus a los profesionales de la salud y otros.

En una reciente revisión sistemática sobre la situación de COVID perinatal ${ }^{9}$ se identificaron 17 publicaciones, la mayoría de China. En estos estudios, en general series de casos, se reportaron datos de SARS-CoV-2 en RN de 214 mujeres embarazadas con infección sospechada o confirmada en el momento del nacimiento. En cuatro RN la RT-PCR para SARS-CoV-2 fue positiva en hisopados respiratorios obtenidos entre las 36 horas y los 8 días después del nacimiento. En esta revisión sistemática otros 3 estudios incluyeron 8 neonatos readmitidos positivos para SARS-CoV-2. La mayoría de los RN tuvo síntomas leves o nulos y no se informó ningún resultado perinatal adverso 9 .

En un estudio de Estados Unidos ${ }^{10}$ se han descrito madres con COVID-19; un escaso número tuvo enfermedad severa o crítica, con porcentajes similares a los descriptos en mujeres no embarazadas, y sin COVID-19 en los RN. Otras revisiones ${ }^{11,12}$ reportaron baja morbilidad y nula mortalidad en madres de diferentes países, ninguna de ellas de América Latina. En una de ellas ${ }^{12}$ la tasa de cesáreas fue del $93 \%$ en 287 casos y en ningún caso se mantuvo la lactancia. Algunos autores han señalado la necesidad de contar con redes de datos que generen evidencia, particularmente en América Latina, a fin de establecer pautas y recomendaciones definitivas adaptadas a la región?.

En este contexto, la Sociedad Iberoamericana de Neonatología (SIBEN) ha convocado a las unidades de América Latina pertenecientes a la Red SIBEN a reportar casos de mujeres embarazadas con infección por SARS-CoV-2 comprobada por RT-PCR con la intención de aportar conocimiento y experiencias sobre la COVID-19 perinatal en esta región.

El objetivo de este estudio fue evaluar y reportar las características clínicas y los resultados de la infección por SARS-CoV-2 en mujeres embarazadas y sus RN en América Latina.

\section{MATERIALES Y MÉTODOS}

Se realizó un estudio descriptivo, mediante reporte prospectivo, convocando a unidades neonatales participantes en la Red SIBEN de América Latina y Guinea Ecuatorial. Se solicitó el reporte de madres con diagnóstico confirmado de COVID-19 mediante RT-PCR para SARS-CoV-2 desde el 6 de marzo al 30 de mayo del 2020. Para cada madre los reportes incluyeron los datos de su RN.

Se registró la presencia o ausencia de síntomas, su gravedad (leve-moderada según la presencia de fiebre o síntomas respiratorios sin requerimiento de oxígeno, o severa en caso de ingreso a terapia intensiva o uso de asistencia respiratoria), y la evolución (vivo o fallecido). Adicionalmente, se registró la prematurez (<37 semanas), la vía de parto, la indicación de aislamiento y el tipo de alimentación del RN (lactancia materna, extracción de leche o uso de fórmulas). Por último, se investigó si la alimentación la realizó la madre, la duración de la estancia hospitalaria y la situación al egreso. Desde el punto de vista ético, todas las unidades de la RED SIBEN firmaron un acuerdo que establecía autonomía y confidencialidad; el envío de datos fue voluntario y desprovisto de variables que permitieran identificar al paciente y se mantuvo la confidencialidad de la unidad donde se originaba el reporte.
Los datos se analizaron según medidas de resumen y frecuencias utilizando valores absolutos y porcentajes para describir la serie.

\section{RESULTADOS}

Se reportaron 86 mujeres embarazadas con COVID-19 confirmadas de 11 unidades de la Red de siete países: Argentina, Colombia, Ecuador, Guinea Ecuatorial, Honduras, Perú y Republica Dominicana.

De ellas, 59 (68\%) fueron asintomáticas; de las 27 (32\%) mujeres con síntomas, 24 (89\%) presentaron síntomas o signos leves o moderados, inclusive fiebre, tos, rinorrea, estornudos, debilidad neuromuscular o radiografía de tórax anormal. Seis mujeres ingresaron a cuidados intensivos; 3 (3,5\%) tuvieron síntomas respiratorios graves, dos recibieron asistencia respiratoria mecánica. Ninguna de las madres falleció.

El $62 \%$ de los partos $(n=53)$ fue por vía vaginal y $38 \%(n=$ 33) fue por cesárea. Ochenta y un embarazos fueron de término (94\%) y $5(6 \%)$ de < 37 semanas de gestación. En 95\% de los casos no se permitió el ingreso de la pareja o de un acompañante a la sala de partos ni acompañamiento durante el puerperio.

A todos los RN de estas 86 madres se les realizó RT-PCR entre las 16 y 36 horas de vida. En 6 (7\%) el resultado del hisopado fue positivo. Todos ellos presentaron dificultad respiratoria leve y transitoria tratada con oxigenoterapia durante 2 a 16 horas, y ninguno falleció.

De los 80 RN con RT-PCR negativa e hijos de madres con RT-PCR positiva para COVID-19, el 90\% $(\mathrm{n}=72)$ fue asintomático. De los 8 con sintomatología neonatal, 6 tuvieron dificultad respiratoria leve transitoria (período transicional feto-neonatal demorado), y 2 fallecieron. Uno de ellos fue un $\mathrm{RN}$ de término con malformación adenomatoidea quística pulmonar que tuvo tres pruebas RT-PCR negativas. El otro fue un RN prematuro extremo que falleció por inmadurez y probable sepsis; sus dos hisopados para RT-PCR fueron negativos.

Para el análisis de alimentación y vínculo madre-hijo se excluyó a los 2 RN que fallecieron y a las 6 madres que ingresaron a cuidado intensivo. De las 78 madres con COVID-19, en $24 \%$ se autorizó a mantener la lactancia materna directa con medidas de protección, en $13 \%$ se extrajo leche para alimentar a su hijo y el resto de los niños fue alimentado con fórmula. Durante la internación no se permitió la permanencia del RN junto a su madre en el 76\% de los casos. En estos RN, la madre no alimentó directamente a su hijo; la leche materna extraída o la fórmula fueron administradas por el personal de salud.

De los 84 RN sobrevivientes, 78 (93\%) fueron dados de alta con su madre. La estancia hospitalaria fue muy variable, con una mediana de 6 días y valores extremos entre 2 y 12 días.

\section{DISCUSIÓN}

Si bien la pandemia de COVID-19 ha ocasionado una preocupación mundial respecto de las consecuencias de la infección viral en las mujeres embarazadas y los RN, en 86 mujeres embarazadas reportadas de 7 países de América Latina a la Red SIBEN no hubo fallecimientos y solamente 3,5\% presentó enfermedad severa. A diferencia de otras publicaciones, los casos presentados en este estudio corresponden a un registro multicéntrico prospectivo de varios países, y no a reportes de una sola institución ni a revisiones de casos de artículos publicados. 
La tasa de cesárea en nuestro estudio es más baja que en las revisiones publicadas, y no se encontraron diferencias entre los RN nacidos por vía vaginal o cesárea. Los hallazgos apoyan las recomendaciones emitidas por asociaciones internacionales en el sentido de que la COVID-19 no es una indicación de cesárea.

Si bien sólo 6 RN de madres con COVID-19 tuvieron infección demostrada por RT-PCR positiva, la toma de la muestra mediante hisopado se realizó entre las 16 y 36 horas de vida. Por ello, es imposible concluir que haya existido transmisión vertical del coronavirus. Como sostienen otros autores ${ }^{8,13,14}$, aún no existe evidencia concluyente que permita establecer la existencia de transmisión vertical en el tercer trimestre. Por otra parte, de los 6 RN con COVID-19 a las pocas horas de nacer, ninguno estuvo gravemente enfermo ni falleció. Los cuadros respiratorios leves que presentaron fueron muy probablemente taquipneas transitorias leves del RN.

Son preocupantes los hallazgos que, en casi todos los casos, no se permitió que las madres tuvieran compañía. Además, es alarmante la muy baja tasa de lactancia materna y la frecuente separación de la díada madre-hijo. En la actualidad todas las sociedades científicas recomiendan que la determinación de separar temporalmente a una madre con COVID-19 de su RN debe hacerse caso por caso y en base a decisiones compartidas. Toda madre tiene derecho a estar con su RN y amamantarlo y en esa situación se aconseja usar estrictas medidas para reducir el riesgo de infección, como barreras físicas (distancia) y uso de máscara facial; cuando eligen amamantar, además, debe llevarse a cabo la higiene de manos y senos antes y después de cada alimentación.

El impacto negativo de estas prácticas para el futuro de la madre y el niño merecerá próximos estudios, pero podrían tener consecuencias relevantes. La depresión posparto y los suicidios $^{15}$, y las disrupciones de las alteraciones vinculares y sus consecuencias ${ }^{15-16}$ ya eran una preocupación en el equipo de salud perinatal antes de la pandemia.

En este estudio se reportan solamente los hallazgos en mujeres embarazadas con COVID-19 confirmada por RT-PCR. En uno de los centros también se realizaron determinaciones de IgG e IgM en más de 300 mujeres embarazadas con sospecha de COVID-19 y sus RN, sin certificación por RT-PCR. Sin embargo, dada la alta posibilidad de falsos positivos o reacción cruzada con otros anticuerpos ${ }^{13,17,18}$, esta información no ha sido incluida en este estudio. Cabe mencionar que en las mujeres con anticuerpos positivos para coronavirus y sin certeza diagnóstica también hubo muy bajas tasas de lactancia materna, elevada separación de la díada madre-hijo y limitación de compañía para la madre.

Durante la respuesta a la COVID-19, los responsables políticos y los profesionales de la salud perinatal deberían considerar en forma prioritaria el riesgo de no identificar adecuadamente los grupos más vulnerables. La atención perinatal y neonatal no debe sufrir durante esta pandemia. Las consecuencias a largo plazo de impedir la lactancia materna y de separar a la madre del RN pueden ser muy dañinas. Como pediatras, neonatólogos y enfermeras neonatales no debemos distraer nuestros esfuerzos de los cuidados humanizados, ya que esto puede tener un impacto negativo grave sobre el bienestar perinatal y la vida futura de las personas.

Afortunadamente, la información preliminar de las instituciones que reportaron los datos de las 86 mujeres embarazadas con COVID-19 y sus RN es muy alentadora; a partir del 11 de mayo ha aumentado significativamente la tasa de lactancia materna y ha disminuido la disrupción vincular madre-hijo en las madres con COVID-19. Es de desear que esto siga mejorando y que todo el equipo de salud reflexione sobre la necesidad de defender el cuidado humanizado y centrado en la familia más allá de la crisis sanitaria y social que vivimos ${ }^{19}$. Hoy más que nunca resulta imperioso sostener recomendaciones específicas para asignar los recursos sanitarios de una manera justa, lo que incluye maximizar los beneficios de las prácticas que se saben efectivas, cuidar la salud de los trabajadores, aplicar la evidencia y colaborar con su construcción a través del reporte de datos, y siempre aplicar los mismos principios éticos para el cuidado de los niños y las madres con COVID-1920 y sin ella.

Concluimos que en esta serie de casos la salud de las madres y sus RN no presentó un impacto muy grave a causa del SARSCoV-2; sin embargo, la pandemia aumenta el riesgo de llevar a cabo prácticas clínicas no basadas en evidencia que atentan contra el cuidado humanizado y centrado en el recién nacido y su familia.

Contribución de los autores. Todos los autores contribuyeron de igual manera a la idea original, el diseño, el análisis e interpretación de los datos y la redacción del manuscrito.

\section{Conflicto de intereses. Ninguno declarado.}

Agradecimientos. A Ylia Espinoza Vivas, Elina Mendoza Ibañez, Manuel Azabache, Verónica Favareto, Cecilia Massache, Odris Tejera, Mary Ramirez Urbina, Irvin Simbarache, Hernando Baquero, María Elena Venegas, Ramiro Alviz, Alejandro Young, Taina Malena por su apoyo con la recopilación de información.

Declaración. Las opiniones expresadas en este manuscrito son responsabilidad de los autores y no reflejan necesariamente los criterios ni la política de la RPSP/PAJPH y/o de la OPS.

\section{REFERENCIAS}

1. Bedford J, Enria D, Giesecke J, Heymann DL, Ihekweazu C, et al. WHO Strategic and Technical Advisory Group for Infectious Hazards. World Health Organization. COVID-19: Towards Controlling of a Pandemic Lancet. 2020;395(10229):1015-18. PubMed PMID: 32197103.

2. Sola, A, Maksimovic L, Montes Bueno MT, Rodríguez S, Cardetti M, Golombek SG, Morgues M. Sociedad Iberoamericana de Neonatología y COVID-19 perinatal: Información y recomendaciones de SIBEN. EDISIBEN. Abril 17, 2020. ISBN 978-1-7923-3225-8.
Disponible en http://www.siben.net/images/files/sibensarscov2 covid19asolav2.pdf Acceso el 30 de mayo de 2020

3. Sola A, García-Alix A, Rodríguez S, Cardetti M, Montes Bueno MT, Arnáez Solís J, Golombek S. Riesgos a la salud materno neonatal en medio de la pandemia viral del covid-19 Declaración Conjunta de SIBEN y Fundación NeNe Disponible en http://www.siben.net/ images/files/sibencovidperinatalsibennenemayo14finalasnpag. pdf Acceso el 30 de mayo de 2020 
4. Arnaez J, Montes MT, Herranz-Rubia N, Garcia-Alix A. The Impact of the Current SARS-CoV-2 Pandemic on Neonatal Care. Front Pediatr. 2020; 8 (247): 1-4. doi: 10.3389/fped.2020.00247.

5. Ma X, Zhu J, Du L. Neonatal management during the coronavirus disease (COVID-19) outbreak: The Chinese experience. NeoReviews. 2020;21(5). https:/ / doi.org/10.1542/neo.21-5-e293 Acceso el 20 de junio de 2020

6. Zaigham M, Andersson O. Maternal and perinatal outcomes with COVID-19: A systematic review of 108 pregnancies. Acta Obstet Gynecol Scand. 2020; 99(7):823-29. doi: 10.1111/aogs.13867. Epub 2020 Apr 20. Acceso el 30 de mayo de2020

7. Mimouni F, Lakshminrusimha S, Pearlman SA, Raju T, Gallagher PG, Mendlovic J. Perinatal aspects on the covid-19 pandemic: a practical resource for perinatal-neonatal specialists. J Perinatol. 2020;40(5):820-26.

8. Chen H, Guo J, Wang C, Luo F, Yu X, Zhang W et al. Clinical characteristics and intrauterine vertical transmission potential of COVID-19 infection in nine pregnant women: a retrospective review of medical records. Lancet. 2020;395: 809-15

9. Duran P, Berman S, Niermeyer S, Jaenisch T, Forster T, Gomez Ponce de Leon $\mathrm{R}$ et al. COVID-19 and newborn health: systematic review. Rev Panam Salud Pública. 2020;44:e54. https://doi.org/10.26633/ RPSP.2020.54

10. Breslin N, Baptiste C, Gyamfi Bannerman C, Miller R, Martinez R, Bernstein $\mathrm{K}$ et al. COVID-19 infection among asymptomatic and symptomatic pregnant women: two weeks of confirmed presentations to an affiliated pair of New York City hospitals. Am J Obstet Gynecol MFM. 2020;2(2):100118. doi: 10.1016/j.ajogmf.2020.100118 Acceso el 30 de mayo de 2020

11. Dashraath P, Wong LJL, Lim MXK et al: Coronavirus Disease 2019 (COVID-19) Pandemic and Pregnancy. Am J Obstet Gynecol. 2020;222(6):521-31. doi:10.1016/j.ajog.2020.03.021

12. Azarkish F, Janghorban R. Impact of COVID-19 infection on maternal and neonatal outcomes: a review of 287 pregnancies. medRxiv. 2020. doi: 10.1101/2020.05.09.20096842. Acceso el 20 de junio de 2020
13. Kimberlin DW, Stagno S. Can SARS-CoV-2 infection be acquired in utero?: more definitive evidence is needed. JAMA. 2020;323(18):1788-9.

14. Fan C, Lei D, Fang C, Li C, Wang M, Liu Y, et al. Perinatal Transmission of COVID-19 Associated SARS-CoV-2: Should We Worry? Clinical Infectious Diseases, ciaa226, https://doi.org/10.1093/cid/ ciaa226 Acceso el 20 de junio de 2020

15. Wisner KL, Sit DK, McShea MC, Rizzo DM, Zoretich RA, et al. Onset Timing, Thoughts of Self-harm, and Diagnoses in Postpartum Women With Screen-Positive Depression Findings. JAMA Psychiatry. 2013;70(5): 490-8

16. Thapa SB, Mainali A, Schwank SE, Acharya G. Maternal mental health in the time of the COVID-19 pandemic. Acta Obstet Gynecol Scand. 2020;99(7):817-8. doi:10.1111/aogs.13894 Acceso el 20 de junio de 2020

17. $\mathrm{Xu} \mathrm{W}, \mathrm{Li} \mathrm{J}, \mathrm{He} X$, et al. The diagnostic value of joint detection of serum IgM and IgG antibodies to 2019-nCoV in 2019-nCoV infection. Chinese J Lab Med. 2020;43(00):E012-E012.

18. Nielsen CM, Hansen K, Andersen HM, Gerstoft J, Vestergaard BF. An enzyme labelled nuclear antigen immunoassay for detection of cytomegalovirus IgM antibodies in human serum: specific and non-specific reactions. J Med Virol. 1987;22(1):67-76

19. Sola A, Sola IP, Rodríguez SP, Golombek SG. Reflections on How to Maintain and Improve Perinatal Care in the Midst of the COVID 19 Pandemic: Let's not Forget Mothers and Newborn Babies! Glob J of Ped \& Neonatol Car. 2(2):2020. GJPNC.MS.ID.000534. doi: 10.33552/ GJPNC.2020.02.000534

20. Emanuel E, Persad G, Upshur R, Thome B, Parker M, Glickman A et al. Fair Allocation of Scarce Medical Resources in the Time of Covid-19. N Engl J Med. 2020;382:2049-55

Manuscrito recibido el 31 de mayo de 2020. Aceptado para publicación, tras revisión, el 30 de junio de 2020.

\section{Perinatal COVID-19 in Latin America}

ABSTRACT Objective. To evaluate and report the clinical characteristics and outcomes of SARS-CoV-2 infection in pregnant women and newborns in Latin America.

Methods. Descriptive study based on the prospective report of the units of the Ibero-American Society of Neonatology Network.

Results. Of 86 pregnant women with COVID-19 confirmed by RT-PCR in seven countries (6 from Latin America, and Equatorial Guinea) 68\% (59) were asymptomatic. Of 32\% of symptomatic women, $89 \%$ (24) had mild symptoms and $3.5 \%$ (3) had severe respiratory symptoms. No women died. The cesarean section rate was $38 \%$; gestational age was < 37 weeks in $6 \%$ of cases. RT-PCR was performed on all newborns between 16 and 36 hours of age; 6 (7\%) were positive. All of them presented mild and transient respiratory distress; none died. Two newborns with negative RT-PCR died from other causes. Breastfeeding was authorized in only $24 \%$ of mothers; in $13 \%$ milk was expressed and $63 \%$ of newborns were fed with formula. In $76 \%$ of cases the motherchild pair was separated, and in $95 \%$ of cases the mother could not be accompanied at delivery or during the postpartum period

Conclusions. The lack of maternal accompaniment, the low rate of breastfeeding and the frequent separation of the mother-child dyad are of concern. The health care team must reflect on the need to defend humanized and family-centered care during this pandemic.

Keywords Coronavirus infections; pandemics; infant, newborn, diseases; infectious disease transmission, vertical; Latin America. 